\title{
The Total Solar Eclipse of 1911
}

\author{
The Moon's Shadow Traces a Course from Australia to Central America
}

\author{
By Frederic R. Honey, Trinity College
}

THE total eclipse of the Sun, which occurs on April 28th of this year, will be observed under somewhat unusual and interesting conditions. The princtpal value of a total solar eclipse is obviously depeñdent upon the possibility of observing the phenomenon from a land area to which it is possible to transport the instruments for observation. In the event of totality occurring in midocean, or in the inaccessible polar regions, the astronomer is deprived of whatever value might accrue to science. But on April 28th an interesting aspect is presented, notwithstanding the fact that totality occurs in midocean; inasmuch as the waters thus visited contain the land areas of the Samoan and Union islands, and the Tonga or Friendly group. Thus is afforded an opportunity for scientific expeditions to these regions, which, though geographically remote, and in some cases must be reached in surf boats, are in no wise discouraging to the enthusiastic observers who will visit them for the purpose of observing the wonderful corona of the Sun. The significance and value of the eclipse phenomena may be judged by the personnel of the several expeditions which are already equipped and on their way to these islands of the equatorial Pacific. The seekers for more light on the science of solar physics are traversing the long distances from such observatory centers as Stonyhurst and South Kensington, England Sydney, Australia; Lick of California and others, for the knowledge which may be obtained in two to four minutes' view of totality; and even these few precious moments of observation are entirely dependent upon fair weather. With all conditions favorable, the patience of the astronomer who has "pursued the shadow" will be rewarded by the wonderful aureole of light which sur rounds the Sun.

The drawings which illustrate this article are designed to show graphically' the relative positions of the Sun, the Earth, and the Moon, at the time of the eclipse.

An eclipse cycle of eighteen years and eleven and one-third days-or a Saros (which means repetition) includes on the average seventy eclipses, of which forty-one are solar, and twenty-nine are lunar. The period from 1893 to 1910 included forty-two period from 1893 to 1910 included forty-two solar and twenty-seven lunar eclipses; and a lunar appulse occurred twice-an average of about four eclipses a year. This year is an excellent illustration of the Saros. Each cluded three years during which no lunar cluded three years during which no lunar
eclipse. has occurred. The years 1857, 1875, eclipse. has occurred. The years 1857,1875,
1893 and 1911 contain no lunar eclipses. A total eclipse of the Sun occured on March 25th, 1857; on April 5th, 1875, on April 16th 1893, and it will be repeated on April 28th, 1911. An annular eclipse occurred on September 17 th, 1857 , on September 29 th, 1875 on October 9th, 1893, and it will be repeated on October 21st, 1911 .

The reason why there will be no lunar eclipse during April or May of the present year will be apparent on an inspection of the plots of the orbits of the Earth and Moon. The latter is magnified eighty times in order to show more clearly the position in order to show more clearly the position
of the Moon relative to the node; and it of the Moon relative to the node; and it
will be noted that in magnifying the orbit, will be noted that in magnifying the orbit, the angle between the radius vector of the Earth's orbit and the line of nodes of the fifteen days before the eclipse of the Sun, when the Moon will be above the plane of when the Moon will be above the plane of
the ecliptic and approaching the descending node $N^{\prime}$, and the radius vector of the Moon's orbit will be projected in the radius vector of the Earth's orbit produced, the Moon will be near the node, but not'near enough to the plane of the ecliptic to come within the Earth's shadow. Also on May 12th, fifteen days after the eclipse, when the Moon will be below the ecliptic, and the radius vector of the Moon's orbit will again be projected in the prolongation of the radius vector of the Earth's orbit, the Moon will be too far away from the plane of the

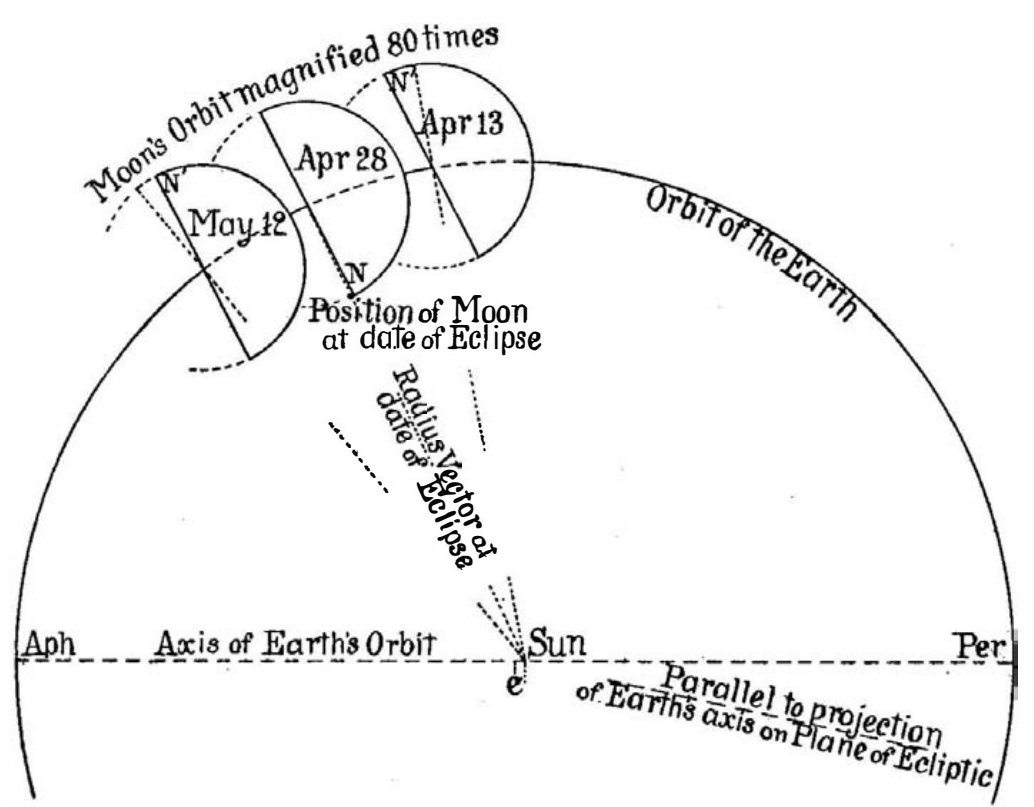

Relative positions of Sun, Moon and Earth at date of eclipse.

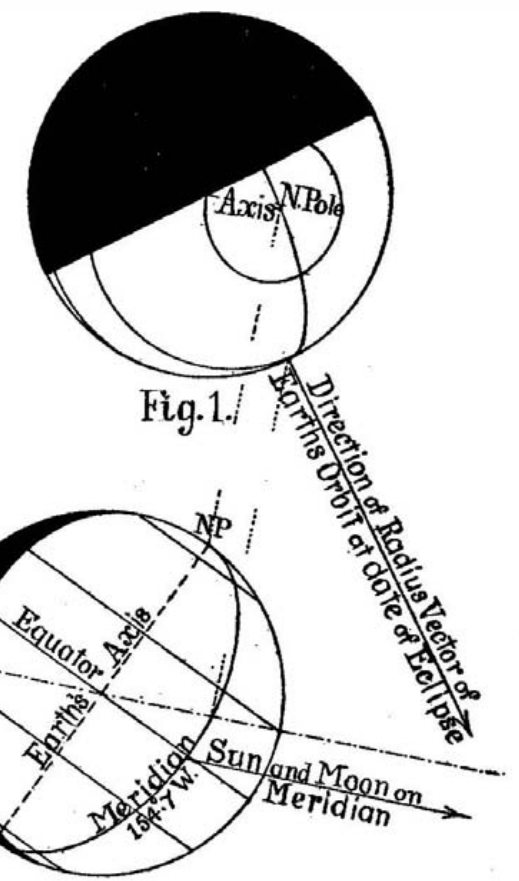

Fig.2.

Position of the Earth and apparent diameters of the Sun and Moon at the time of the eclipse.

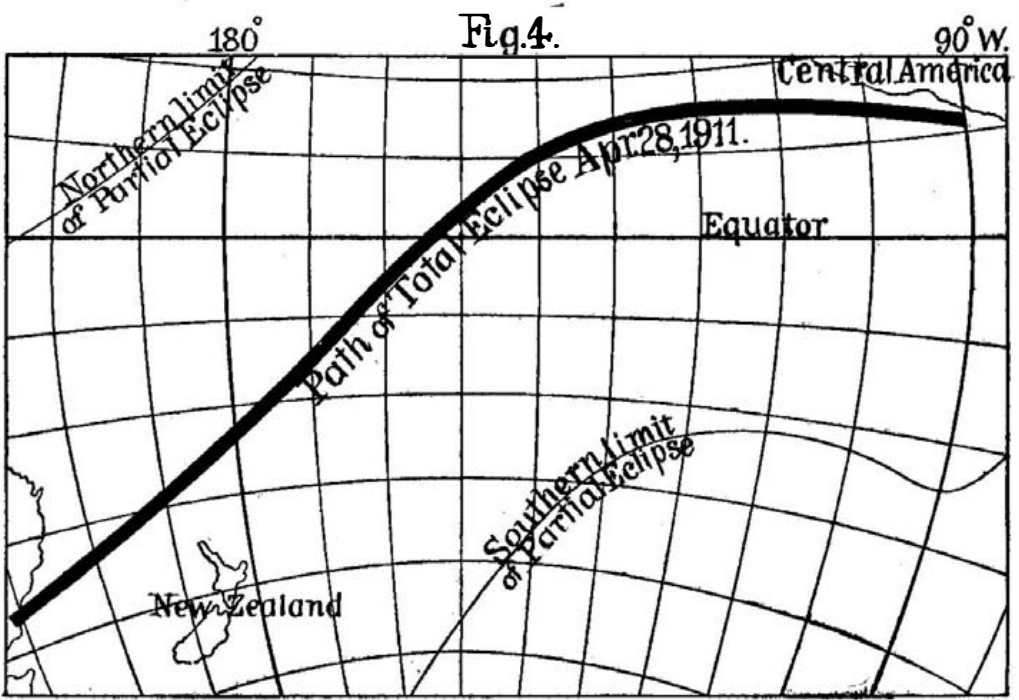

Path traced by the Moon's shadow.

point from which the central eclipse will be visible at noon, are shown in both projections. The point from which the central eclipse will be visible at noon will be very near the equator (lat. 0.6 deg. S.; long. 154.7 deg. W.), and will be below the plane of the ecliptic corresponding to the situation shown in the plot of the Earth's orbit. The Sun will be totally eclipsed, and the proportion between the apparent diameters of the Sun and Moon at the time of the eclipse is shown in Fig. 3. At each successive Saros the Moon's shadow is projected on a different part of the Earth's surface farther west. This is on account of the additional fraction of onethird of a day during which the Earth rotates into a new position. But during the interval of fifty-four years and thirty-four days (March 25th, 1857, to April 28th, 1911) or three times eighteen years and eleven and one-third days, the Earth will have made a number of rotations which will result in the Moon's shadow being projected on very nearly the same part of the Earth's surface. The date of the total eclipse in 1857 was March 25th $10.41 \mathrm{~h}$. That of 1911 will be April 28th, $10.27 \mathrm{~h}$. within a fraction of an hour of three times the length of an eclipse period.

Fig. 4 shows the path of the total eclipse which extends from Australia across the equator to Central America. As a partial eclipse it will be visible in the eastern part of Australia and New Guinea, and will include Van Dieman's Land, New Zealand, and the islands to the north. It will be visible in the United States, Mexico, and Central America. Washington will be very near the limit of visibility, where a small partial eclipse will be seen, the Sun setting eclipsed.

\section{A Novel Method of Obtaining Pure} Oxygen

THE chemical laboratory of the National Bureau of Standards recently had need of a large quantity of practically pure oxygen gas in carrying out certain work. This gas was prepared in one of the physical laboratories of that institution by the electrolysis of water. The positive electrode was suspended in an ordinary round quart bottle with its bottom removed. This bottle was provided with a hollow metal stopper which formed part of the electrical connection between the positive electrode and the outside main. The electrolytic apparatus was placed outside the building, and as the hydrogen given off at the negative pole was not needed, it: was allowed to go to waste.

The oxygen gas escaping through the hollow stopper under a pressure of only a. few inches of water was conducted through a long copper tube to a purifier containing platinized quartz. Although this purifier was used constantly no impurities were found in the gas. From the purifier the gas was conducted through a valve-head to a two-liter cylinder of heavy brass lined with nickel. This cylinder was suspended in a bath of liquid air at atmospheric: pressure. Fresh made liquid air boils at about 9 deg. C., lower temperature than oxygen, and this difference was sufficient to cause the oxygen to liquefy. When the brass cylinder was filled with liquid oxygen, the current was shut off from the electrolytic apparatus, a common steel flask was connected up with the valve head, the liquid air bath removed, and the liquid oxygen at once began to vaporize owing to the heat received from the surrounding atmosphere. When the gage on the valve-

farther from the node, the Moon's shadow would be projected wholly on the southern hemisphere. Fig. 1 is the projection of the Earth on the plane of the ecliptic, and Fig. 2 its projection on a plane which is parallel to its axis and perpendicular to the "plane of the ecliptic. The equator, the tropics, the polar cir cles, and that meridian which passes through the head indicated sufficient pressure the valves were losed, and another flask was substituted for the filled one. The oxygen thus obtained was found more than 99.9 per cent pure. It will be seen that oxygen produced in this way is as near absolute purity as is possible. It is an expensive process, however, and cannot compete with the liquid air rectification process. 